\title{
Electronic properties of GaAs surfaces etched in an electron cyclotron resonance source and chemically passivated using $\mathbf{P}_{\mathbf{2}} \mathbf{S}_{\mathbf{5}}$
}

\author{
O. J. Glembocki and J. A. Tuchman ${ }^{\text {a) }}$ \\ U.S. Naval Research Laboratory, Washington, DC 20375-5347 \\ J. A. Dagata ${ }^{\text {b) }}$ \\ National Institute of Standards and Technology, Gaithersburg, Maryland 20899 \\ K. K. Ko and S. W. Pang \\ The University of Michigan, Ann Arbor, Michigan 48109 \\ C. E. Stutz \\ Wright-Patterson Laboratories, Dayton, Ohio 45433
}

(Received 24 March 1998; accepted for publication 6 May 1998)

\begin{abstract}
Photoreflectance has been used to study the electronic properties of (100) GaAs surfaces exposed to a $\mathrm{Cl}_{2} / \mathrm{Ar}$ plasma generated by an electron cyclotron resonance source and subsequently passivated by $\mathrm{P}_{2} \mathrm{~S}_{5}$. The plasma etch shifts the Fermi level of $p$-GaAs from near the valence band to midgap, but has no effect on $n$-GaAs. For ion energies below $250 \mathrm{eV}$, post-etch $\mathrm{P}_{2} \mathrm{~S}_{5}$ chemical passivation removes the surface etch damage and restores the electronic properties to pre-etch conditions. Above $250 \mathrm{eV}$, the etch produces subsurface defects which cannot be chemically passivated. Auger electron spectroscopy shows that etching increases As at the GaAs/oxide interface, while passivation reduces it. (C) 1998 American Institute of Physics. [S0003-6951(98)04127-8]
\end{abstract}

The formation of modern day three-dimensional electronic devices often involves the removal of material through etching. As device dimensions approach submicron scales, exposed surfaces become important in the electronic properties of the device. Fermi-level pinning at the exposed surfaces leads to charge depletion and consequently a degradation of device performance.

It is well known that dry etching of GaAs, even at the low voltages used in electron cyclotron resonance (ECR) etching can damage the surface significantly. In addition, GaAs surfaces etched by ECR and exposed to ambient conditions have As rich GaAs/oxide interfaces and Fermi levels heavily pinned at midgap. ${ }^{1}$ This type of damage has a significant effect on barrier heights when Schottky contacts are made on etched surfaces ${ }^{2}$ and on the free electron concentration in GaAs wires. ${ }^{3}$

It has been shown that chemical passivation with $\mathrm{P}_{2} \mathrm{~S}_{5}$ can improve the electronic properties of ambient GaAs surfaces. ${ }^{4-6}$ This treatment has been shown to produce a uniform, 1-2 monolayer thick oxide, which is Ga rich and resistant to any further oxidation. ${ }^{6}$ In addition, Glembocki et al. showed that $\mathrm{P}_{2} \mathrm{~S}_{5}$ passivation improves the photovoltaic response of (100) $n$-GaAs that was processed by chemically assisted ion beam etching. ${ }^{7}$ Because $\mathrm{P}_{2} \mathrm{~S}_{5}$ removes As from $\mathrm{GaAs}$ oxides, it may heal ECR related surface damage and reduce the Fermi-level pinning.

In this study, we used photoreflectance of special GaAs multilayer structures grown by molecular beam epitaxy (MBE) to examine the effects of etching with a $\mathrm{Cl}_{2} / \mathrm{Ar}$ plasma generated by an ECR source and subsequent chemi-

\footnotetext{
${ }^{a}$ Present address: Optex Corporation, Gaithersburg, MD 20899.

${ }^{b)}$ Also with: Precision Engineering Division, Manufacturing Engineering Laboratory, Technology Division, U.S. Department of Commerce.
}

cal passivation on the Fermi-level pinning of the (100) GaAs/oxide interface. Auger electron spectroscopy was used to identify the chemical composition of the interface.

The structures used in this study were $1500 \AA$ A thick undoped $\left(p=1 \times 10^{14} \mathrm{~cm}^{-3}\right)$ GaAs layers that were grown by MBE on $n^{+}$or $p^{+}$buffer layers and will be referred to as UN $\left(n^{+}\right.$buffer) or UP ( $p^{+}$buffer). The buffer layers were doped to $1-2 \times 10^{18} \mathrm{~cm}^{-3}$ and were $1.0 \mu \mathrm{m}$ thick. Solutions of Poisson's equation show that this configuration produces a constant electric field, $F$, in the undoped layer, which is directly related to the surface potential $V_{s}$, by $F=V_{s} / d$, where $d$ is the insulator thickness. The constant field and high quality of the undoped layers leads to photoreflectance spectra which have many Franz-Keldysh oscillations (FKOs), allowing us to accurately determine the built-in field. Similar types of structures were recently used in various studies of GaAs surfaces. ${ }^{7-13}$

The photoreflectance (PR) apparatus used in this study is similar to those described previously in the literature. ${ }^{14}$ The probe light consisted of a monochromatic beam created by passing white light from a $60 \mathrm{~W}$ quartz halogen lamp through a SPEX 1/4 m monochromator. The PR pump beam was a $0.25 \mathrm{~mW}$ green $(5430 \AA) \mathrm{HeNe}$ laser mechanically chopped at $393 \mathrm{~Hz}$. The monochromatic, reflected light was detected with a Si photodiode and analyzed by a lock-in amplifier.

Two sets of UN and UP samples were used in the experiments, with one set serving as an unprocessed control. All pairs of samples were $1 \mathrm{~cm}^{2}$ neighbors from the same region of a larger wafer. PR measurements from all of the samples prior to etching showed variations in surface electric fields of less than $5 \%$ across a wafer.

The etching was performed in a plasma generated by an ECR source on top of a radio frequency (rf)-powered elec- 
trode. The source was a multipolar plasma disk source surrounded by 12 permanent magnets, and driven at $2.45 \mathrm{GHz}$. The ion energy was independently controlled by a 13.56 $\mathrm{MHz}$ rf power supply. The distance between the sample stage and the ECR source was adjustable between 7 and 27 $\mathrm{cm} . \mathrm{Cl}_{2}$ gas was introduced into the etch chamber through a gas ring situated $1 \mathrm{~cm}$ below the wafer stage, while Ar gas was introduced radially through the base of the ECR source.

The ion energy was varied by changing the rf power. The sample to source distance was $15 \mathrm{~cm}$ and the $\mathrm{Cl}_{2} / \mathrm{Ar}$ mixture had $30 \% \mathrm{Cl}_{2}$ at a pressure of $0.6 \mathrm{mTorr}$ and a microwave power of $50 \mathrm{~W}$. Increases in rf power from 20 to $300 \mathrm{~W}$ produced changes in $\left|V_{\mathrm{dc}}\right|$ from 77 to $484 \mathrm{~V}$. In all cases, the etch depth was kept constant at $\sim 350 \AA$.

The ex situ $\mathrm{P}_{2} \mathrm{~S}_{5}$ surface passivation treatments were performed after etching, using the procedure described by Dagata et al. ${ }^{6}$ The etched sample was treated for $7 \mathrm{~min}$ in a $50{ }^{\circ} \mathrm{C}$ solution of $\left(\mathrm{NH}_{4}\right)_{2} \mathrm{~S}$ and then washed for $7 \mathrm{~min}$ in a $50{ }^{\circ} \mathrm{C}$ dilute, aqueous solution of $\mathrm{P}_{2} \mathrm{~S}_{5} /\left(\mathrm{NH}_{4}\right)_{2} \mathrm{~S}$. All of the samples were rinsed in de-ionized water after the chemical passivation treatment was completed.

Photoreflectance spectra were taken before and after each processing step (etching and passivation). Auger electron spectroscopy was performed on a control piece and its processed neighbor.

Photoreflectance spectroscopy measures the built-in electric field in a semiconductor because a constant applied electric field modifies the band edge of a semiconductor in a manner predicted by the Franz-Keldysh theory. For the case of Lorentzian broadening and ignoring excitonic effects, the PR line shape is represented by Airy functions which for photon energies greater than the band gap can be asymptotically written as: ${ }^{15}$

$$
\begin{aligned}
E^{2}\left(E-E_{g}\right) \frac{\Delta R}{R} \sim & V_{p} \cos \left[\frac{4}{3}\left(\frac{E-E_{g}}{\hbar \theta}\right)^{3 / 2}+\phi\right] \\
& \times \exp \left[-\left(\frac{E-E_{g}}{\hbar \theta)^{3}}\right)^{1 / 2}\right],
\end{aligned}
$$

where $E$ is the photon energy, $E_{g}$ is the energy gap, $\Gamma$ is lifetime broadening, $\hbar \theta$ is the electro-optic energy, and $V_{p}$ is the applied ac voltage in the region of the electric field. In photoreflectance, the ac voltage, $V_{p}$, is just the ac photovoltage induced by the pump laser. The quantity $\hbar \theta$ is related to the electric field, $\mathbf{F}$, and the reduced interband effective mass along the direction of the electric field, $\mu_{\|}\left[=\left(\mu_{e}^{-1}\right.\right.$ $\left.\left.+\mu_{h}^{-1}\right)^{-1}\right]$, through $\hbar \theta=\left(e^{2} F^{2} \hbar^{2} / 2 \mu_{\|}\right)^{1 / 3}$. Because Eq. (1) exhibits a periodic behavior its Fourier transform will yield the period and hence the electric field through $\hbar \theta$. We have used this procedure to determine the electric field from the FKO.

Shown in Fig. 1 are $300 \mathrm{~K}$ photoreflectance spectra for unetched (a) and etched (b) and $\mathrm{P}_{2} \mathrm{~S}_{5}$ passivated (c) UP samples. The etch conditions consisted of a microwave power of $50 \mathrm{~W}$ and a dc bias of $250 \mathrm{~V}$. In each spectrum we see FKO because of the electric field in the undoped layer. ECR etching increases the period of the FKO indicating a larger surface field. This increase in field can be caused by two effects: a reduction in the thickness of the undoped region and an increase in the surface potential. A post-etch

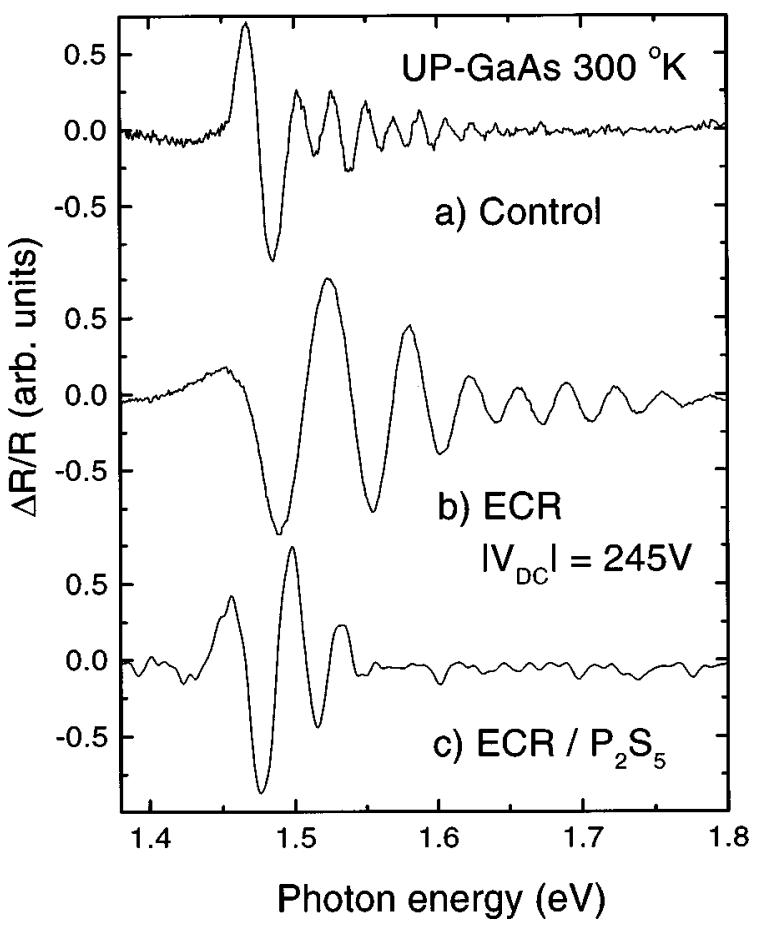

FIG. 1. Photoreflectance spectra for a UP sample that was (a) unetched, (b) ECR etched with a dc bias of $\left|V_{\mathrm{DC}}\right|=245 \mathrm{~V}$, and (c) post-etch passivated with $\mathrm{P}_{2} \mathrm{~S}_{5}$.

measurement of the etch depth indicates that the predominant change in the field is due to a change in the Fermi-level pinning position. We find that $V_{f}$ changed from $0.3 \mathrm{eV}$ prior to etching to $0.65 \mathrm{eV}$ after etching. This motion of the Fermi level has been attributed to the formation of excess As in at the GaAs/oxide interface of the etched samples.

It has previously been shown that $\mathrm{P}_{2} \mathrm{~S}_{5}$ passivation is an effective vehicle for removing excess As in GaAs oxides. This should allow us to remove the excess As in the etched samples and to restore the Fermi level to pre-etch conditions. The data of Fig. 1(d) show the effects of $\mathrm{P}_{2} \mathrm{~S}_{5}$ passivation on the PR spectrum of the etched sample. We see that the FKO decreases dramatically indicating a reduced field. From the period of the FKO and the thickness of the undoped layer, we obtain a Fermi-level pinning position $(0.36 \mathrm{eV})$ similar to that of the unetched UP sample. We conclude that the passivation reverses the surface induced etch damage.

The results above suggest that the passivation removes excess As from the GaAs/oxide interface of the etched samples. To verify this, Auger spectroscopy was performed on an unetched control sample and one that was etched with a dc bias of $\left|V_{\mathrm{dc}}\right|=375 \mathrm{~V}$ and subsequently passivated using $\mathrm{P}_{2} \mathrm{~S}_{5}$. Data were taken through the native oxide, as well as after Ar ion sputtering of the native oxide. In the unsputtered control and ECR etched samples, the intensity of the oxygen Auger peaks was the same, indicating similar amounts of oxygen in both. We found $\mathrm{Ga} / \mathrm{As}$ ratios of 1.3, 1.0, and 1.5 for the control, the etched, and the etched/passivated samples, respectively, suggesting that the stoichiometry of the oxides was different. After sputter removal of the oxide, all of samples had Ga/As ratios near 1.6, which is characteristic of sputtered, bulk GaAs. This indicates that the nature of the oxide and its interface with GaAs is different for the three cases. These data also show that the position of the 


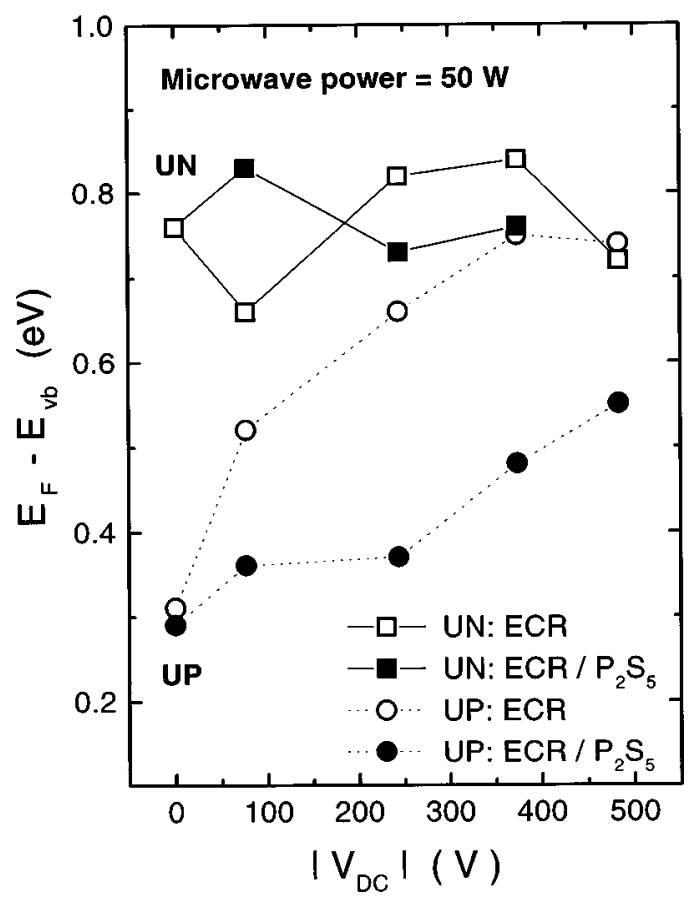

FIG. 2. The Fermi-level pinning position of the etched UN (squares) and UP (circles) samples as a function of self-induced dc bias. The open symbols refer data from etched samples, while the solid symbols correspond to data from samples that were etched and passivated. All energies are relative to the top of the valence band (VB).

Fermi level in $p$-GaAs is controlled by excess As at the GaAs/oxide interface.

In Fig. 2 we show the room temperature Fermi-level positions for the various UN and UP samples as a function of the self-induced bias, $\left|V_{\mathrm{dc}}\right|$. The Fermi level positions of the unetched samples agree well with previously reported data for (100) GaAs. ${ }^{8,11}$ We see that with increasing ion energy, the Fermi level position of the UP sample moves away from the valence band and stabilizes near midgap. This behavior has previously been ascribed to the formation of etch induced damage that results in an As rich oxide. Figure 2 shows that the chemical passivation is very effective in restoring the Fermi level to pre-etch conditions for ion energies below $250 \mathrm{~V}$.

Above $250 \mathrm{~V}$ the Fermi level of the passivated samples is a function of ion energy. This suggests that the etch induced damage is no longer confined to the top 1 or 2 monolayers, which are modified by the chemical passivation. The data above $250 \mathrm{~V}$ can be understood in terms of subsurface damage by remembering that we extract the Fermi-level position from the measured electric field. Because the test layer is undoped, we use $F=V_{s} / d$, where $F$ is the measured field. If on the other hand the ions created subsurface defects whose density decreased as $(d-z)^{n}$, where $d$ is the depletion width, then the relationship between the field and surface potential would become $F=[(n+2) /(n+1)]\left(V_{s} / d\right)$. Note that $n=0$ yields $F=2 V_{s} / d$, which is the correct result for a uniformly doped layer. With this, and $n=1$ and $n=0$ we can account for the 375 and $500 \mathrm{~V}$ data, respectively. In both cases, the surface potential that we obtain is $V_{s}=0.33 \mathrm{~V}$, in good agreement with the other passivated samples. This analysis suggests that the $\mathrm{P}_{2} \mathrm{~S}_{5}$ passivation produces the same surface in all cases and that the etch above $250 \mathrm{~V}$ produces subsurface damage in the form of charged defects whose range increases with ion energy. Note that the difference between the $n=0$ and $n=1$ damage profiles is that the $n$ $=1$ profile drops linearly from the surface, while the $n=0$ one is uniform. This is not unreasonable, since previous damage studies have shown that even at low ion energies, the damage can penetrate many tens of nanometers. ${ }^{3,12}$

The Fermi-level position of the UN samples as a function of ion energy (Fig. 2) is hardly effected, even at high dc biases. For ion energies below $250 \mathrm{~V}$, this behavior can be attributed to the fact that midgap pinning of $n$-GaAs is difficult if not impossible to change. ${ }^{16}$ Above $250 \mathrm{~V}$, where subsurface damage is formed in the UP samples, we see no evidence of the damage in the UN samples. This can be explained if we assume that the etch induced subsurface defect is amphoteric and that its energy lies between the valence band and midgap. ${ }^{12}$ For $n$-GaAs, the state is a deep donor that remains neutral when the Fermi level is above it. For $p-\mathrm{GaAs}$ the defect is an acceptor and is negative when the Fermi level is above it. This accounts for the fact that the undoped layer in the UN structure remains insulating, but in the UP samples, it behaves as if it were doped.

In conclusion, we have shown that ECR etching produces both surface and subsurface damage. The surface damage is due to excess As at the GaAs/oxide interface and can be healed with a etch post $\mathrm{P}_{2} \mathrm{~S}_{5}$ chemical passivation, which removes the excess As. The passivation studies revealed the existence of additional subsurface damage for ion energies greater than $250 \mathrm{eV}$. The damage has been attributed to bulk defects whose energy levels lie in the lower half of the band gap and are active only in $p$-GaAs. These results show the nature and extent of etch induced damage in ECR etching of GaAs.

${ }^{1}$ O. J. Glembocki, J. A. Tuchman, K. K. Ko, S. W. Pang, A. Giordana, R. Kaplan, and C. E. Stutz, Appl. Phys. Lett. 66, 3054 (1995).

${ }^{2}$ K. K. Ko and S. W. Pang, J. Electrochem. Soc. 141, 255 (1994).

${ }^{3}$ K. K. Ko, S. W. Pang, T. Brock, M. W. Cole, and L. M. Cassas, J. Vac. Sci. Technol. B 12, 3382 (1994).

${ }^{4}$ H. H. Lee, R. J. Racicot, and S. H. Lee, Appl. Phys. Lett. 54, 724 (1989).

${ }^{5}$ K. C. Hwang and S. S. Li, Appl. Phys. Lett. 67, 2162 (1990).

${ }^{6}$ J. A. Dagata, W. Tseng, J. Bennett, J. Schneir, and H. H. Harary, Appl. Phys. Lett. 59, 3288 (1991); Ultramicroscopy 42-44, 1288 (1992).

${ }^{7}$ O. J. Glembocki, J. A. Dagata, E. E. Dobisz, and D. S. Katzer, Mater. Res. Soc. Symp. Proc. 236, 217 (1992).

${ }^{8}$ F. H. Pollak, J. Vac. Sci. Technol. B 11, 1710 (1993); see also J. M. Woodall, Mater. Res. Soc. Symp. Proc. 324, 141 (1994).

${ }^{9}$ X. Yin, H. M. Chen, F. H. Pollak, Y. Cao, P. A. Montano, P. D. Kirchner, G. D. Pettit, and J. M. Woodall, J. Vac. Sci. Technol. B 9, 2114 (1991).

${ }^{10}$ X. Yin, H. M. Chen, F. H. Pollak, Y. Cao, P. A. Montano, P. D. Kirchner, G. D. Pettit, and J. M. Woodall, J. Vac. Sci. Technol. B 10, 131 (1991).

${ }^{11}$ O. J. Glembocki, J. A. Dagata, E. S. Snow, and D. S. Katzer, Appl. Surf. Sci. 63, 143 (1993).

${ }^{12}$ H. Nakanishi, K. Wada, and W. Walukiewicz, J. Appl. Phys. 78, 5103 (1995).

${ }^{13}$ M. Lopez-Lopez, M. Melendez-Lira, and S. Goto, Appl. Phys. Lett. 71, 338 (1997).

${ }^{14}$ O. J. Glembocki, Proc. SPIE 1286, 1 (1990).

${ }^{15}$ D. E. Aspnes, Phys. Rev. 153, 972 (1967); R. N. Bhattacharya, H. Shen, P. Parayanthal, F. H. Pollak, T. Coutts, and H. Aharoni, Phys. Rev. B 37, 4044 (1988).

${ }^{16}$ D. Yan, F. H. Pollak, T. P. Chin, and J. M. Woodall, Phys. Rev. B 52, 4674 (1995). 